\title{
EXPERIMENTAL TIME RESOLVED ELECTRON BEAM TEMPERATURE MEASUREMENTS USING BREMSSTRAHLUNG DIAGNOSTICS ${ }^{*}$
}

\author{
P.R. Menge, J.E. Maenchen, M.G. Mazarakis, S.E. Rosenthal \\ Sandia National Laboratories, PO Box 5800, Albuquerque, New Mexico 87185-1193
}

Abstract

Electron beam temperature, $\beta_{\perp}(=\mathrm{v} \perp / \mathrm{v})$, is important to control for the development of high dose flash radiographic bremsstrahlung sources. At high voltage (> $5 \mathrm{MV}$ ) increasing electron beam temperature has a serious deleterious effect on dose production. The average and time resolved behavior of beam temperature was measured during radiographic experiments on the HERMES III accelerator (10 MV, $50 \mathrm{kA}, 70 \mathrm{~ns})$. A linear array of thermoluminescent dosimeters (TLDs) were used to estimate the time integrated average of beam temperature. On and off-axis photoconducting diamond (PCD) detectors were used to measure the time resolved bremsstrahlung dose rate, which is dependent on beam energy and temperature. The beam temperature can be determined by correlating PCD response with accelerator voltage and current and also by analyzing the ratio of $\mathrm{PCD}$ amplitudes on and off axis. This ratio is insensitive to voltage and current and thus, is more reliable than utilizing absolute dose rate. The data is unfolded using comparisons with Monte Carlo simulations to obtain absolute beam temperatures. The data taken on HERMES III show abrupt increases in $\beta \perp$ midway through the pulse indicating rapid onset of beam instability.

\section{EFFECT OF $\beta_{\perp}$ ON X-RAY DOSE}

The successful application of Inductive Voltage Adders (IVAs) to the development of high dose $(\approx \mathrm{kRad}$ at $1 \mathrm{~m})$ flash $\mathrm{x}$-ray radiography requires the production of high current, high voltage electron beams while maintaining low divergence (low beam temperature) [1]. The effect of beam temperature on dose can be seen in Figure 1. This graph shows that at $10 \mathrm{MV}$ the on-axis dose is reduced by $60 \%$ for an e-beam going from cold to $\beta_{\perp}=0.3\left(17^{\circ}\right.$ divergence). These curves were compiled from several Monte Carlo [2] runs varying the electron beam temperature at 5 and $10 \mathrm{MV}$. It is, therefore, important to know the beam temperature in experiment in order to compare expected and measured dose, verify physical understanding of the radiographic diode, and examine possible instabilities of which increased $\beta_{\perp}$ is a symptom.

\section{II. $\beta \perp$ MEASUREMENTS ON HERMES III}

Time integrated and time resolved beam temperatures were measured on radiographic source experiments on the
HERMES III accelerator ( $10 \mathrm{MV}, 50 \mathrm{kA}, 70 \mathrm{~ns}$ ) [3]. The purpose of these experiments was to prove the utility of

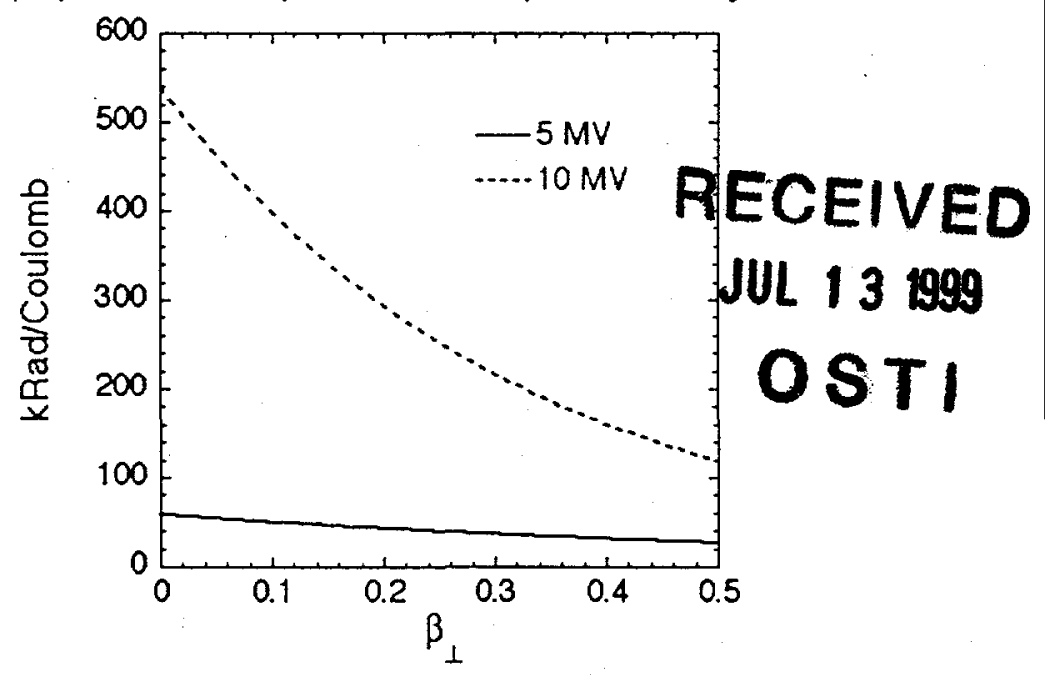

Figure 1. Effect of beam temperature on on-axis dose. Abscissa is dose normalized with beam charge at $1 \mathrm{~m}$ from the source. Ordinate is average beam temperature in units of $\beta_{\perp}\left(v_{\perp} / v\right)$.

IVAs as a possible $x$-ray source driver for DOE's future Advanced Hydrotest Facility (AHF). By immersing a small cathode needle $(0.5 \mathrm{~mm}$ dia) along a strong solenoidal field (up to $50 \mathrm{~T}$ ) coupled to a tantalum anode (bremsstrahlung converter), a small $x$-ray spot producing high dose $(\approx 1 \mathrm{~mm}$ and $\mathrm{kRad}$ at $1 \mathrm{~m})$ is believed possible. Beam temperature was determined through analysis of the $x$-ray output measured by time integrated thermoluminescent detectors (TLDs) and time resolved PCDs [4].

\section{A. Time Integrated $\beta_{\perp}$ Measurements}

A spatial array of TLDs was fielded to measure the angular dose profile of the $x$-ray field. The array consisted of 26 TLDs covering a $21-\mathrm{cm}$ line crossing the radiographic axis and placed $22 \mathrm{~cm}$ from the bremsstrahlung converter, thus covering $\pm 25^{\circ}$. Figure 2 compares the experimental dose profile for a typical shot, \#4693, with profiles predicted by Monte Carlo simulations of the experiment at $10 \mathrm{MV}$. The profiles indicate a time resolved average beam temperature of slightly more than $\beta_{\perp}=0.2$. The curves are not smooth due to occultation by structures in the diode and other

\footnotetext{
* Sandia is a multi-program laboratory operated by Sandia Corporation, a Lockheed-Martin company, for the United States Department of Energy, under contract DE-AC04-94AL85000
} 


\section{DISCLAIMER}

This report was prepared as an account of work sponsored by an agency of the United States Government. Neither the United States Government nor any agency thereof, nor any of their employees, make any warranty, express or implied, or assumes any legal liability or responsibility for the accuracy, completeness, or usefulness of any information, apparatus, product, or process disclosed, or represents that its use would not infringe privately owned rights. Reference herein to any specific commercial product, process, or service by trade name, trademark, manufacturer, or otherwise does not necessarily constitute or imply its endorsement, recommendation, or favoring by the United States Government or any agency thereof. The views and opinions of authors expressed herein do not necessarily state or reflect those of the United States Government or any agency thereof. 


\section{DISCLAIMER}

Portions of this document may be illegible in electronic image products. Images are produced from the best available original document. 
surrounding fixtures. The main concern when analyzing this data is that one must assume the beam has a constant energy ( $10 \mathrm{MV}$ in this case), in reality the angular profile changes with electron energy. Also, this TLD measurement gives no information on how the beam temperature changes during the pulse. It is believed that due to these two reasons, the TLD profile does not exactly match $\beta_{\perp}=0.2$ or 0.3 nor can it be matched to an interpolation between the two Monte Carlo calculations which are mono-energetic.

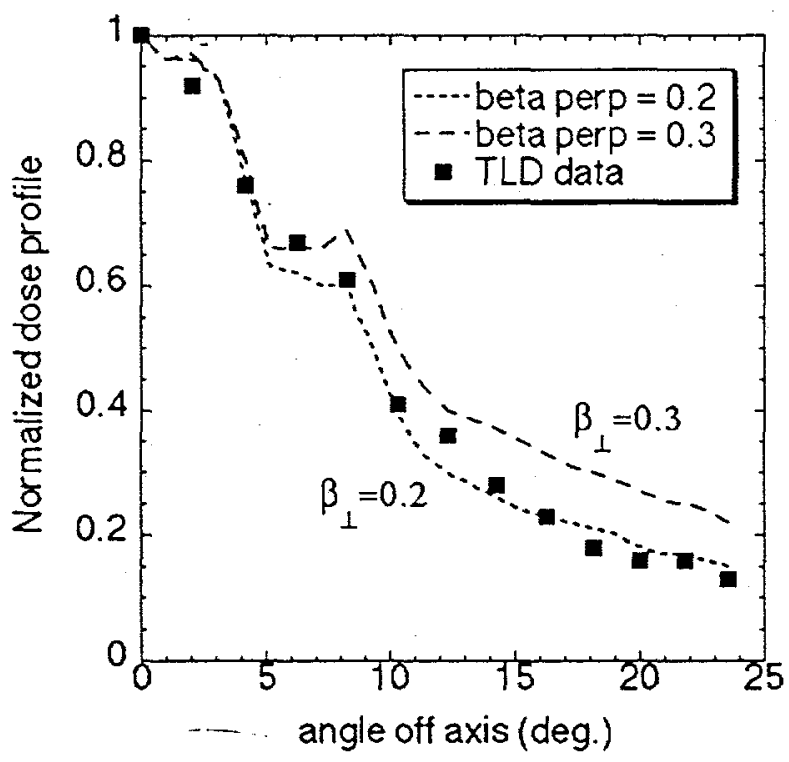

Figure 2. Graph showing HERMES III experimental data and Monte Carlo predictions for the $x$-ray dose angular profile.

\section{B. Time Dependent $\beta_{\perp}$ Measurements}

The $x$-ray dose rate is dependent on the voltage. current and temperature of the beam. If voltage and current are known then the beam temperature can be determined. Figure 3 illustrates the dependence of on-axis dose rate on voltage and $\beta_{\perp}$. This graph was made from 42 Monte Carlo runs simulating the HERMES III experiment varying voltage and $\beta \perp$ (one voltage and one $\beta_{\perp}$ per run). The dose is at $1 \mathrm{~m}$. This surface fits the equation:

$$
\dot{D}=340 \pi V^{3.2} \operatorname{Exp}\left[\frac{-(V+0.5) \beta_{\perp}}{3.45}\right]
$$

where $D$ is dose rate in rad/s at $1 \mathrm{~m}, I$ is beam current in $A$, and $V$ is beam voltage in MV. Thus if $V, I$, and dose rate are known at any given time $\beta_{\perp}$ can be calculated. This was routinely done through use of PCDs, which are fast hard radiation detectors.

Another method used to calculate time dependent $\beta_{\perp}$ is through comparison of the dose rate on and off-axis. Inspection of Figure 2 indicates that the ratio of dose (or dose rate) at, for example, $15^{\circ}$ to on-axis $\left(0^{\circ}\right)$ is expected to be different for $\beta_{\perp}$ s of 0.2 or 0.3 . In general, at any voltage as $\beta_{\perp}$ increases the ratio of off to on-axis dose approaches one. This is true because as forward energy decreases, the forward peaking of the bremsstrahlung decreases, and the source becomes more and more isotropic. Figure 4 graphs this ratio of off to on-axis dose rate versus $\beta_{\perp}$ predicted by Monte Carlo for several voltages spanning $6-12 \mathrm{MV}$.

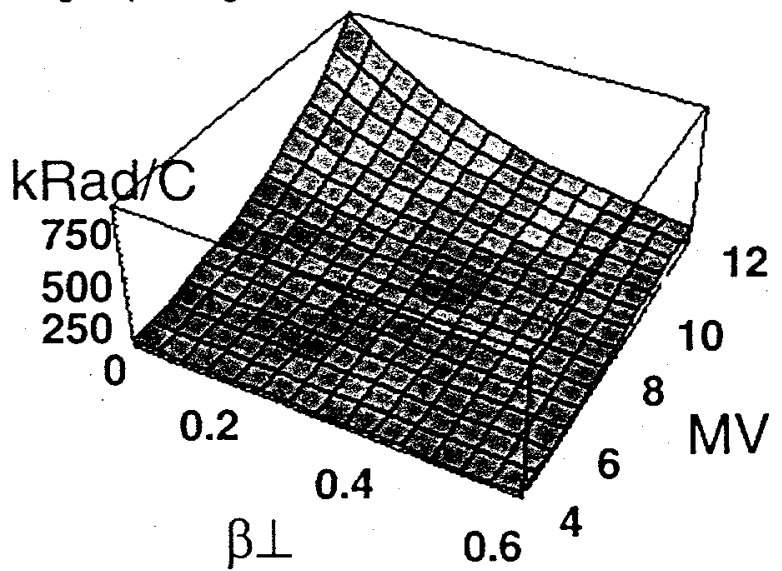

Figure 3. Monte Carlo dose rate predictions varying voltage and beam temperature.

The off-axis point used in this graph is $15^{\circ}$. Note that this ratio is not particularly sensitive to voltage in this range. Thus, this method of computing beam temperature is more appealing than that suggested by equation 1 , since voltage need be known less accurately and current not at all.

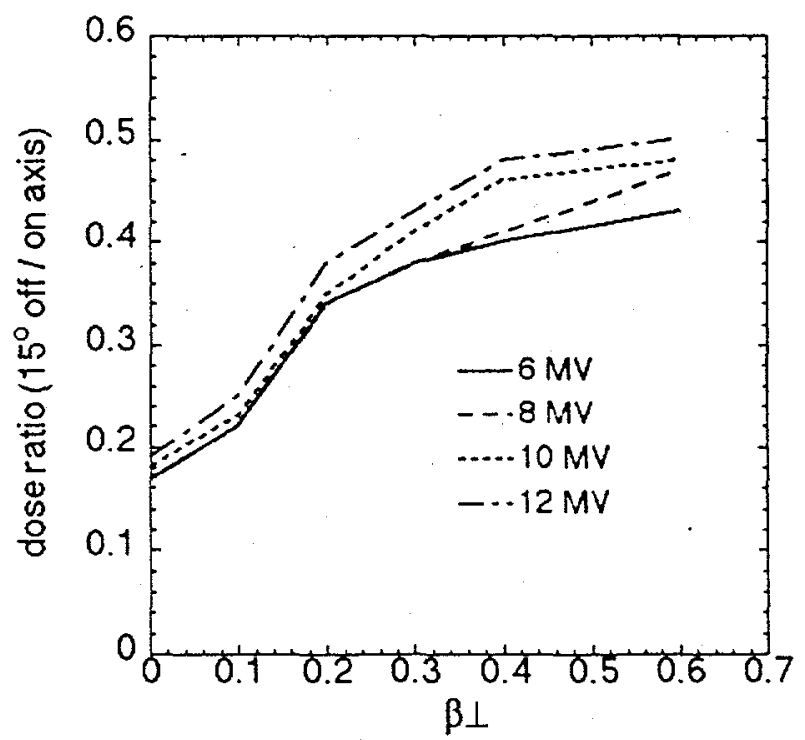

Figure 4. Ratio of off to on-axis dose rate vs. $\beta_{1}$ for several voltages achievable by HERMES III. The closeness of the curves indicates insensitivity to voltage. 
Measuring voltage on magnetically insulated transmission lines (MITLs) like HERMES III is most easily done through measuring the total and boundary currents and applying the formula:

$$
V=Z_{f} \sqrt{I_{t}^{2}-I_{b}^{2}}
$$

where $\mathrm{V}$ is voltage, $\mathrm{Z}_{\mathrm{f}}$ is the flow impedance (approximately equal to the vacuum impedance for HERMES III in negative polarity), $I_{t}$ is the total (anode) current, and $I_{b}$ is the boundary (cathode) current [5].

Determining voltage accurately in this experiment depends on an accurate measurement of currents and is further hampered by an inability to measure boundary current close to the diode. This inability is caused by the need for a plastic flashover switch inserted on the cathode stalk just upstream of the diode to eliminate prepulse, but it does not allow diagnostic cables any further downstream. Therefore, diode voltage must be inferred using inductive corrections to voltages calculated upstream of the diode.

Figure 5 shows an example of time dependent $\beta_{\perp}$ analysis using both methods. The calculated diode voltage and measured current for shot \#4693 are shown in part a) and the PCD signals and $\beta_{\perp}$ data unfolds are shown in part b). The thick (green) line is $\beta \perp$ calculated from equation 1 (method 1 ), while the thinner (red) line is $\beta_{\perp}$ calculated from PCD signal ratios on-axis and $15^{\circ}$ off-axis (method 2). The PCD signals themselves are shown as the dotted lines. Note the relative agreement between the two methods and the rapid increase in $\beta_{\perp}$ beginning near $40 \mathrm{~ns}$. This increase drastically reduces the output dose of the pulse. Analysis of time resolved pinhole photographs of the beam spot size indicate rapid spot blooming corresponding to the time of increasing $\beta_{\perp}$. The pinhole photographs and their timing relative to Figure 5 is shown in Figure 6. These photos were taken with an electro-optical x-ray camera with a "shutter speed" of 2 ns for each frame. Note that starting with photo 3 taken at $40 \mathrm{~ns}$, the beam begins to blow up and lose its confinement by the magnetic field.
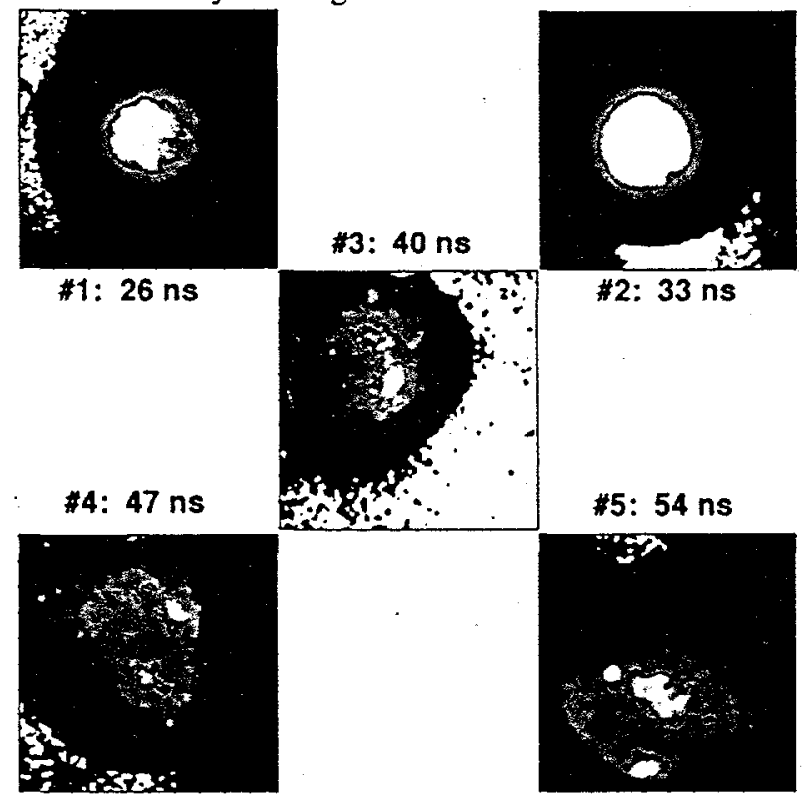

Figure 6. X-ray framing camera photographs of the $\mathrm{x}$-ray source. The frames are $7.5 \mathrm{~mm} \times 7.5 \mathrm{~mm}$.
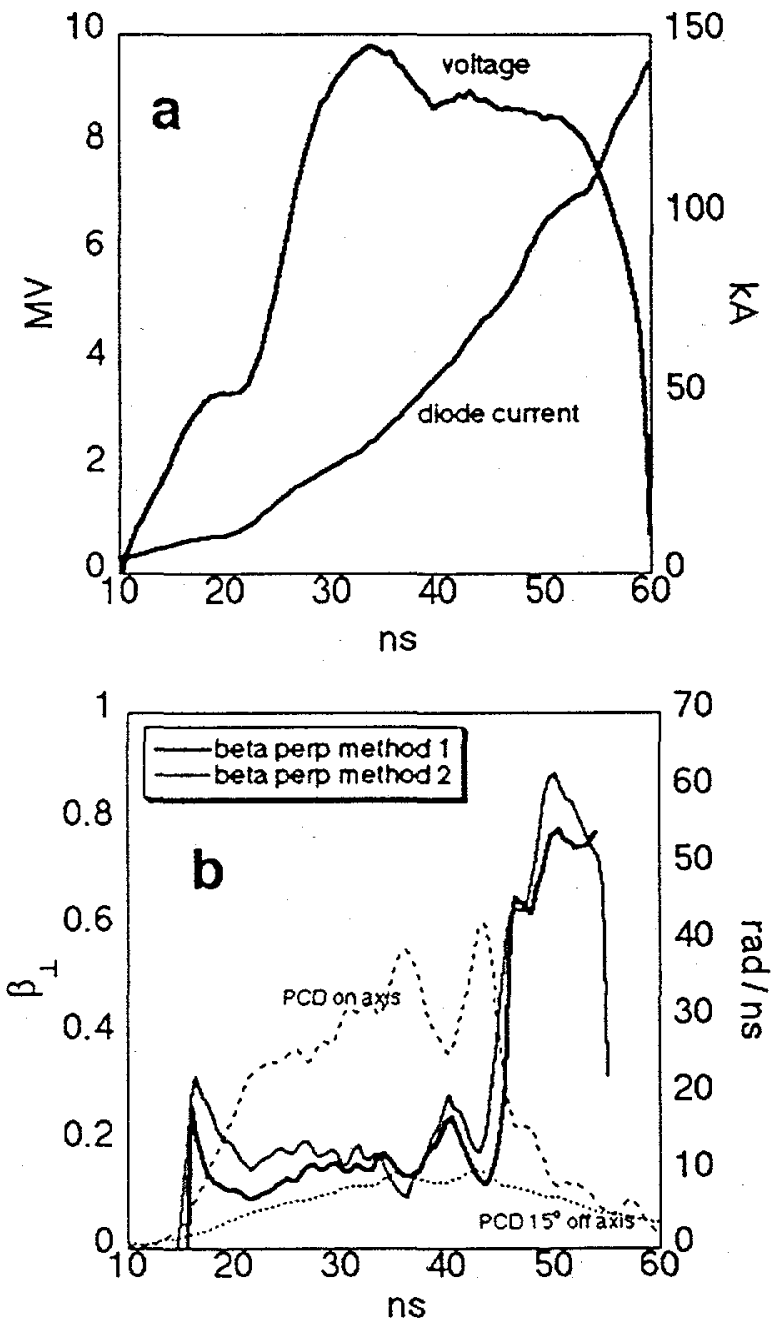

Figure 5. a) Voltage calculated from eqn. 2 and diode current for shot 4693 . b) $\beta_{\perp}$ calculated from eqn. 1 using voltage and current from a) (thick green line), $\beta_{\perp}$ calculated from Figure 4 using off and on-axis PCDs (thin red line), and the dose rate from PCDs on and off-axis (dotted lines).

This behavior is indicative of the onset of an enhanced ion-hose instability [6], which can arise in these types of MITL driven high current density, magnetically immersed diodes. Suppression of this instability is currently a major focus of this program.

In summary, methods to determine time integrated and time-resolved electron bearn temperature have been developed for high voltage radiographic accelerators. These methods rely on Monte Carlo simulations of the experiment to develop models of dose and dose rate as a function of voltage, current, and angle. Rapid increases in beam temperature during the pulse are commonly seen and correspond to degradation of dose rate and beam spot confinement. Onset of an enhanced ion hose instability can explain the results. 


\section{REFERENCES}

[1] John Maenchen, et. al. "Inductive Voltage Adder Driven X-Ray Sources For Hydrodynamic Radiography", these proceedings.

[2] J.A. Halbleib, R.P. Kensek, T.A. Mehlhorn, G.D. Valdez, S.M. Setzer, and M.J. Berger, IEEE Tran. Nuc. Sci. 39, 1025 (1992).

[3] J.J. Ramirez et. al. "The HERMES-III Program", Proc. of the $6^{\text {th }}$ IEEE Pulsed Power Conference, Arlington Virginia, June 29 - July 1, 1987.

[4] R.S. Wagner, et. al., Proc 1992 IEEE Nucl. Sci. Symposium 1, 88 (1992).

[5] C.W. Mendel, et. al., J. Appl. Phys., 71, 3731 (1992)

[6] D.R. Welch, R.E. Clark, T.P. Hughes, B.V. Oliver, "Theoretical Support for the Inductive-Voltage-Adder Radiography Program - at Sandia National Laboratories", Mission Research Report \#MRC/ABQ-R-1889, Sept. 1998. 